MRS Advances (C) 2017 Materials Research Society

DOI: 10.1557/adv.2017.492

\title{
Polyethersulfone/Graphene Oxide Ultrafiltration Membranes from Solutions in Ionic Liquid
}

Dinesh. K. Mahalingam, DooLi. Kim and Suzana. P. Nunes

King Abdullah University of Science and Technology (KAUST), Biological and Environmental Science and Engineering Division (BESE), 23955-6900 Thuwal, Saudi Arabia

\begin{abstract}
Novel high flux polyethersulfone (PES) ultrafiltration membranes were fabricated by incorporating different amounts of graphene oxide (GO) sheets to PES as nanofillers. The membranes were prepared from solutions in 50/50 1-ethyl-3-methylimidazoliumdiethylphosphate/N,N-dimethyl formamide. It was observed that the water permeance increased from 550 to $800 \mathrm{~L} \mathrm{~m}^{-2} \mathrm{~h}^{-1} \mathrm{bar}^{-1}$, with incorporation of $1 \mathrm{wt} \% \mathrm{GO}$, keeping a molecular weight cutoff (MWCO) of approximately 32-34 kg mol${ }^{-1}$. Cross-sectional scanning electron microscopy images of GO/PES membranes showed the formation of ultrathin selective layer unlike pristine membranes. Contact angle measurements confirmed the increase of hydrophilicity, by increasing the GO concentration. The rejection of humic acid and bovine serum albumin was demonstrated. The mechanical properties were improved, compared with the pristine membranes. The performance was just above the trade-off relationship between permeance and separation factor for PES membranes reported in the literature.
\end{abstract}

\section{INTRODUCTION}

The use of ionic liquids as solvent or co-solvent for the preparation of membranes has been explored by our group[1-4] and others[5], motivated by two aspects: (i) their low toxicity[2] and low vapor pressure, compared to solvents currently used in the industry for membrane manufacture, and (ii) their capacity of inducing different morphologies, guided by hydrogen bond and electrostatic interactions[6]. We prepared flat sheet cellulose membranes by dissolving in 1-ethyl-3-methylimidazolium acetate ([EMIM]OAc)[1], hollow fiber membranes of polyacrylonitrile from solution in dimethyl sulfoxide/([EMIM]OAc[4] and of cellulose acetate in acetone/([EMIM]OAc[3], and hydroxyl-polytriazole in dimethyl carbonate/[EMIM]OAc[7]. PES is one of the most common membrane materials in the membrane industry for ultrafiltration and as support for reverse osmosis[8] with application in desalination, waste water treatment, biotechnology and pharmaceutical industries, oil-water separation, protein mixture separation, food industry and other filtration processes. While being an attractive material in membrane filtration, PES is known to be a hydrophobic polymer and is highly susceptible to fouling, without surface modification. We recently prepared PES membranes from solutions in 1-ethyl-3methylimidazolium diethylphosphate ([EMIM]DEP)[2] with molecular weight cut-off (MWCO) varying from 1.3 to $30 \mathrm{~kg} \mathrm{~mol}^{-1}$ and permeance values from 20 to $140 \mathrm{~L} \mathrm{~m}^{-2} \mathrm{~h}^{-1} \mathrm{bar}^{-1}$, respectively. So far we only used ionic liquid for membranes fully constituted by organic polymers or block copolymers. However there is a large potential to explore casting solutions containing nanofillers such as graphene oxide (GO), which could be dispersed and eventually aligned under the influence of ionic liquids. GO, as two-dimensional carbon nano sheet decorated with oxygencontaining functional groups, such as hydroxyl and carboxyl groups, has growing interest for 
membrane application[9], as thin selective layer for water transport or as additive to improve mechanical properties, reduce fouling[10] or add further functionalization to the membrane[11,12]. Preparation, structure and reactivity of GO were reviewed in detail by Dreyer et al[13]. Herein, we used ionic liquid based solvent mixtures to dissolve polymers and disperse GO to obtain hybrid membranes. We demonstrate it for GO/PES and anticipate that ionic liquids could be a useful solvent for tuning morphology of other 2D-nanofillers/polymer mixed-matrix membranes.

\section{EXPERIMENT}

\section{$\underline{\text { Materials }}$}

Polyethersulfone (PES) (Ultrason®, BASF, average Mn = 75 000), 1-ethyl-3methylimidazolium-diethylphosphate ([EMIM]DEP, $\geq 98.0 \%$, Sigma-Aldrich) and N,Ndimethylformamide (DMF) ( $\geq 99.8 \%$, Sigma-Aldrich) were used for membrane fabrication. Polyethylene glycol (PEG) from Sigma-Aldrich with molecular weights of 0.3, 1.5, 6, 10, 35, and $100 \mathrm{~kg} \mathrm{~mol}^{-1}$ were used for the solute rejection test and the calculation of the molecular weight cut-off (MWCO, minimum molecular weight with $90 \%$ rejection). Graphite flakes were purchased from Sigma-Aldrich and used as received. Sulphuric acid $\left(\mathrm{H}_{2} \mathrm{SO}_{4}, 97 \%\right)$, hydrochloric acid $\left(\mathrm{HCl}, 35 \%\right.$ in water), hydrogen peroxide $\left(\mathrm{H}_{2} \mathrm{O}_{2}, 30 \%\right.$ in water), potassium permanganate $\left(\mathrm{KMnO}_{4}\right)$ were procured from Sigma Aldrich. Bovine serum albumin (BSA) was supplied by Sigma Aldrich.

\section{Graphene oxide (GO) synthesis}

GO was prepared from natural graphite flakes by a modified Hummer's method[14]. In a typical procedure, $2 \mathrm{~g}$ of graphite flakes were mixed into a round-bottomed flask containing 250 $\mathrm{ml}$ of $\mathrm{H}_{2} \mathrm{SO}_{4}$ and the resulting mixture was stirred for $1 \mathrm{hr}$ at $25^{\circ} \mathrm{C}$. About $10 \mathrm{~g}$ of $\mathrm{KMnO}_{4}$ were added to the mixture under stirring until a viscous dark-green mixture was obtained and stirring was continued for $24 \mathrm{~h}$ at $25^{\circ} \mathrm{C}$. The mixture was transferred into an ice bath and a mixture containing $100 \mathrm{ml}$ of $\mathrm{H}_{2} \mathrm{O}_{2}$ and $500 \mathrm{ml}$ of deionized (DI) water was added slowly. The color of the solution mixture turned to golden yellow after the addition of $\mathrm{H}_{2} \mathrm{O}_{2}$ and DI water. The resulting mixture was stirred for $2 \mathrm{~h}$ and centrifuged at $10000 \mathrm{rpm}$ to remove impurities. Thereafter, the solution was treated with $10 \% \mathrm{HCl}$ and centrifuged thrice with $10 \% \mathrm{HCl}$. Subsequently, $\mathrm{HCl}$ treated oxidized exfoliated graphite particles were washed and centrifuged with DI water until the solution $\mathrm{pH}$ turns $\geq 5$. The obtained dark brown GO paste was freezedried and stored dry.

\section{Fabrication of asymmetric GO/PES membranes}

GO nanosheets were homogeneously dispersed in DMF and [EMIM]DEP via ultrasonication. The following concentration was chosen for membrane fabrication: $18 \mathrm{wt} \% \mathrm{PES}$, different GO concentrations $(0 ; 0.5 ; 1$; and $2 \mathrm{wt} \%$, relative to the polymer) in 50/50 
DMF/[EMIM]DEP solvent mixtures. Thereafter, PES was dissolved in the homogeneously dispersed solution containing $\mathrm{GO}$ at $90{ }^{\circ} \mathrm{C}$ for a day. The obtained polymer solutions were then cast on a glass plate using a casting knife with a $200 \mu \mathrm{m}$ gap and immersed into DI water to induce phase separation.

\section{Membrane Characterization}

The surface and cross-section morphologies were analyzed using Field Emission Scanning Electron Microscope (FESEM, FEI Nova Nano). The membranes were freeze-dried to avoid pore collapse and sputter-coated with iridium.

Water permeance and rejection of neutral solutes (PEG) were investigated using a stirred dead-end ultrafiltration cell set up under 2 bar pressure, as reported before[2].

The MWCO was measured by filtering a mixture of PEG molecules with different molecular weights dissolved in water. Then, the feed and permeate concentrations were analyzed by Gel Permeation Chromatography (GPC) with PL aquagel-OH 40 and 60 columns, (1260 infinity GPC/SEC, Agilent technologies). The rejection of humic acids was estimated by measuring solute absorption at $254 \mathrm{~nm}$ using UV-vis spectroscopy.

The strain-stress curves were investigated by Dynamic Mechanical Analysis (DMA, TA Instruments DMA-Q800) equipped with a film tension clamp. Experiments were carried out at $25{ }^{\circ} \mathrm{C}$ with controlled force ramp of $0.05 \mathrm{~N} \mathrm{~min}^{-1}$ and a preload force of $0.001 \mathrm{~N}$. The membrane thickness was measured with a Mitutoyo-Absolute micrometer and ProMax-Fowler caliper.

Contact angles $(\theta)$ were measured by Attension Theta optical Tensiometer (KSV instruments) at room temperature. Each sample was measured at 5 different spots and the average value was reported.

\section{DISCUSSION}

Figure 1 shows the surface and cross-section morphologies of prepared GO/PES hybrid membranes with 0, 0.5, 1 and $2 \mathrm{wt} \%$ GO from solutions in DMF/[EMIM]DEP. The cross-section of pristine membranes exhibited a thick dense sponge-like top-layer over a porous supporting layer with finger-like macro voids. GO incorporation into polymer matrix changed membrane sub layer by practically eliminating the dense sponge layer. A very thin skin layer was obtained.

The surface morphology was devoid of microscopic defects with smooth surfaces and not much change was detected after GO modification in the membranes. The only difference was the change in porosity after the addition of GO. The cross section of the pristine membranes exhibited a thick dense top-layer followed by a porous supporting layer with finger-like macro voids. On the other hand, the GO incorporation into the polymer matrix changed the membrane sub layer by eliminating the dense layer and thus a very thin skin layer was obtained. The exchange between solvent and non-solvent during phase inversion process becomes rapid with the addition of hydrophilic fillers in the matrix of the membranes. This is because of the presence of hydrophilic groups decorated on GO edges and basal planes.

A homogeneous casting solution was prepared to cast all membranes. The addition of GO into polymer gradually changed membrane color from white to dark brown. This is an indication 
that GO is dispersed in polymer matrix homogeneously. Additionally, introduction of GO involves in structural changes, which is revealed in cross sectional SEM images.

It was shown that oxygen containing groups, structural defects, edge-to-edge slits and non-oxide regions of GO nanosheets provided channels with higher selectivity and transport rate towards water molecules[1].

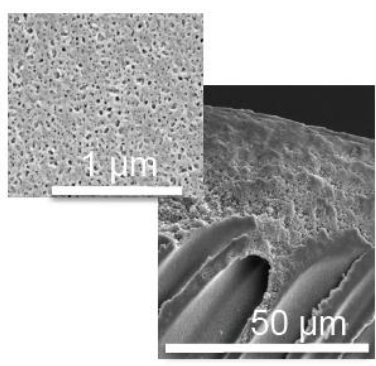

(a)

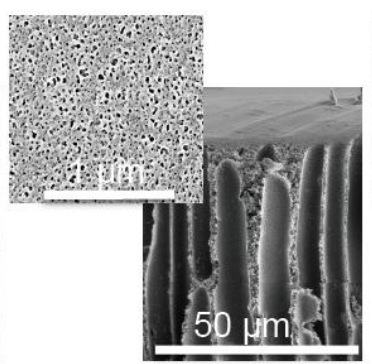

(b)

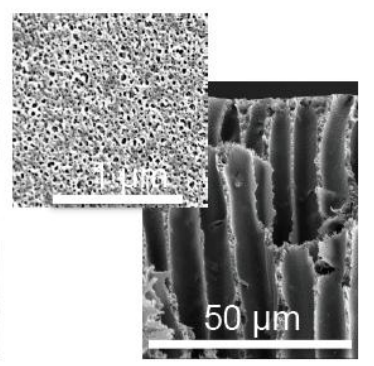

(c)

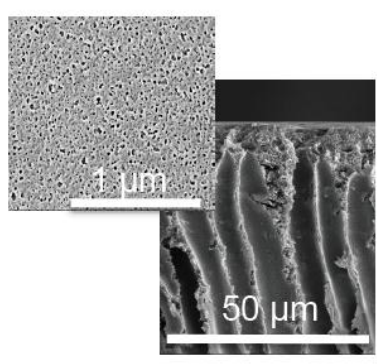

(d)

Figure 1. SEM images of surfaces and cross-sections of GO/PES hybrid membranes with different GO weight fractions: (a) 0, (b) 0.5 , (c) 1 , (d) $2 \mathrm{wt} \%$.

The water permeance of GO/PES membranes was measured as shown in Figure 2. It can be seen that the water permeance for hybrid membranes increased with increasing GO concentration up to $1 \mathrm{wt} \%$. The maximum water permeance was $803 \mathrm{~L} \mathrm{~m}^{-2} \mathrm{~h}^{-1} \mathrm{bar}^{-1}$ for the membrane with $1 \mathrm{wt} \%$ GO loading. The water permeance decreased with further loading of GO. The increase in the water permeance can be explained on the basis of enhanced membrane hydrophilicity and decrease of the skin layer thickness. The maximum water permeance can be correlated with the contact angle results, shown in Figure $2 b$, which is inversely proportional to the hydrophilicity. Functional groups such as hydroxyl, carboxyl, carbonyl in the GO surface are responsible for improving the hydrophilicity The membranes with $2 \mathrm{wt} \%$ GO might have suffered aggregation, not having GO as dispersed as in the case of $1 \mathrm{wt} \%$.

We did not find any appreciable chemical change. Imidazolium-based ionic liquids might exchange cations with negatively charged $\mathrm{COOH}$ groups in $\mathrm{GO}$ and interacts through weak $\pi-\pi$ stacking interactions with GO, enhancing compatibility between GO and ionic liquid. 


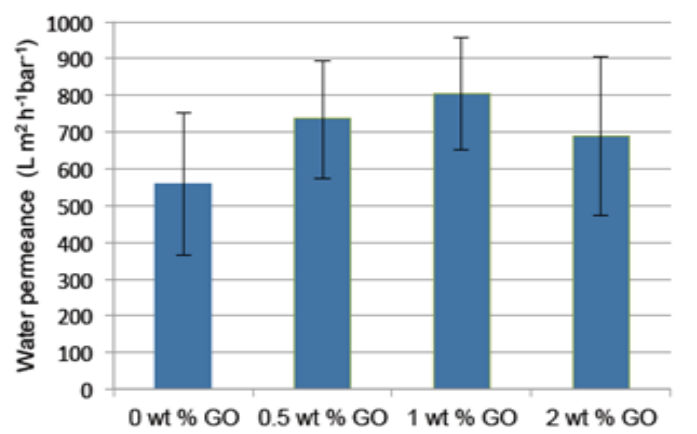

(a)

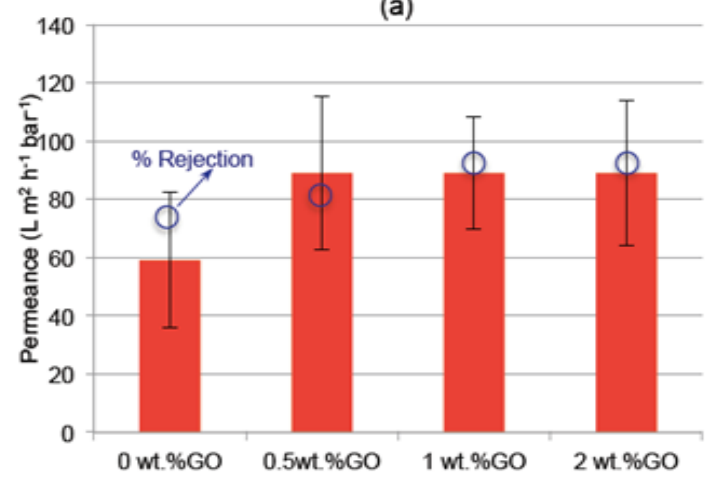

(c)

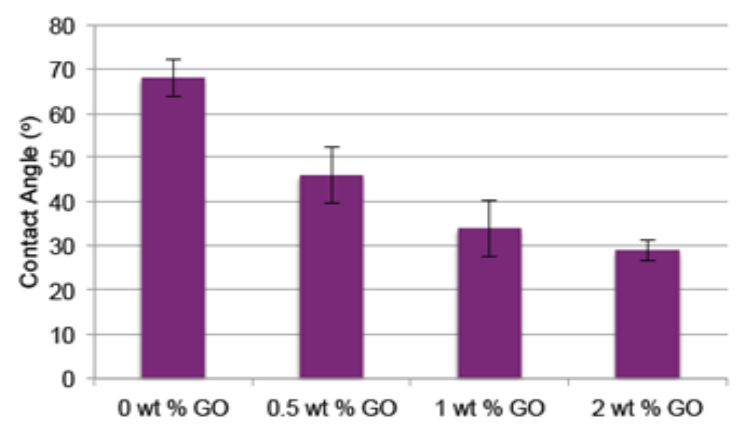

(b)

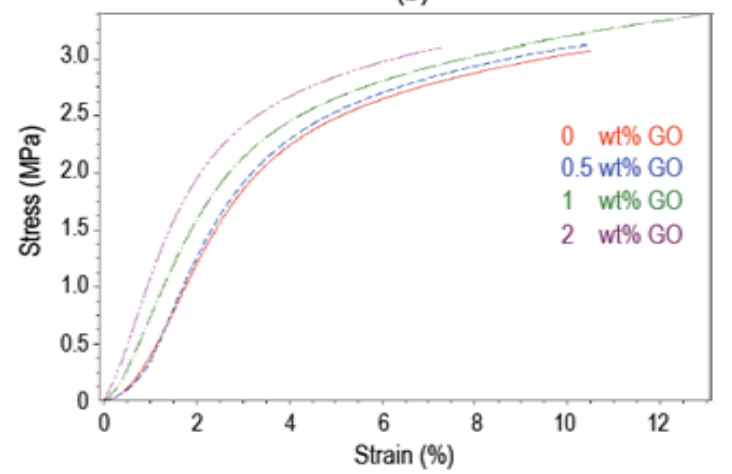

(d)

Figure 2. Properties of GO/PES membranes: (a) pure water permeance at 2 bar and $400 \mathrm{rpm}$, (b) contact angle, (c) permeance and rejection of aqueous solutions containing humic acids, (d) stress-strain curves.

The MWCO's of GO/PES membranes were similar, varying from 32 to, 38, 34 and 31 $\mathrm{kg} / \mathrm{mol}$, as the GO wt $\%$ increases from 0 to $0.5,1$ and $2 \mathrm{wt} \%$. All membranes rejected almost $100 \%$ albumin $(66.5 \mathrm{~kg} / \mathrm{mol})$ and could be useful to separate it from solutes with molecular weight lower than $30 \mathrm{~kg} / \mathrm{mol}$.

The permeance and rejection of humic acid solutions depended on the amount of GO, as shown in Figure 2c. The permeance was $90 \mathrm{~L} \mathrm{~m}^{-2} \mathrm{~h}^{-1} \mathrm{bar}^{-1}, 50 \%$ higher than for the pristine membrane. Further addition of GO did not increase the flux. The thin selective layer of the membranes, containing ultrafine channels with regularity, greatly influences ultrafiltration performance. The incorporation of GO changes the membrane microstructure. The removal efficiency of humic acid and BSA increased substantially with the incorporation of GO, which, is ascribed to the negative charge behavior of hybrid membranes. Due to the addition of GO, membranes become negatively charged that involves in the electrostatic repulsions between negatively charged membrane and humic acid and BSA. Additionally, enhanced hydrophilicity on the resultant membrane surface through polar interactions suppressed hydrophobic interactions between BSA and membrane surface.

The BSA selectivity was evaluated and compared to other PES membranes reported in the literature, as shown in Figure 3, in analogy to the selectivity - permeance trade-off curve published by Mehta and Zydney[15]. The separation factor was calculated from equation 1.

$\mathrm{S}_{\mathrm{a}}=\mathrm{S}_{0} /\left(1-\mathrm{S}_{0}\right) \exp \left(\mathrm{J}_{\mathrm{v}} / \mathrm{k}\right)+\mathrm{S}_{0}$ 
where, $S_{a}$ is the separation factor or selectivity for the membrane, $S_{0}$ is the sieving coefficient calculated from $\left(S_{0}=C_{\text {filtrate }} / C_{f e e d}\right), J_{v}$ is the volumetric filtrate flux (volume flow rate per membrane area) and $k$ is the mass transfer coefficients. To avoid highly polarized conditions $J_{v} / k$ was considered to be less than 10[15]. The GO/PES membranes have high separation factors, compared to other available membranes and have permeance at least as high as the best values reported for the range of $30 \mathrm{~kg} \mathrm{~mol}^{-1} \mathrm{MWCO}$.

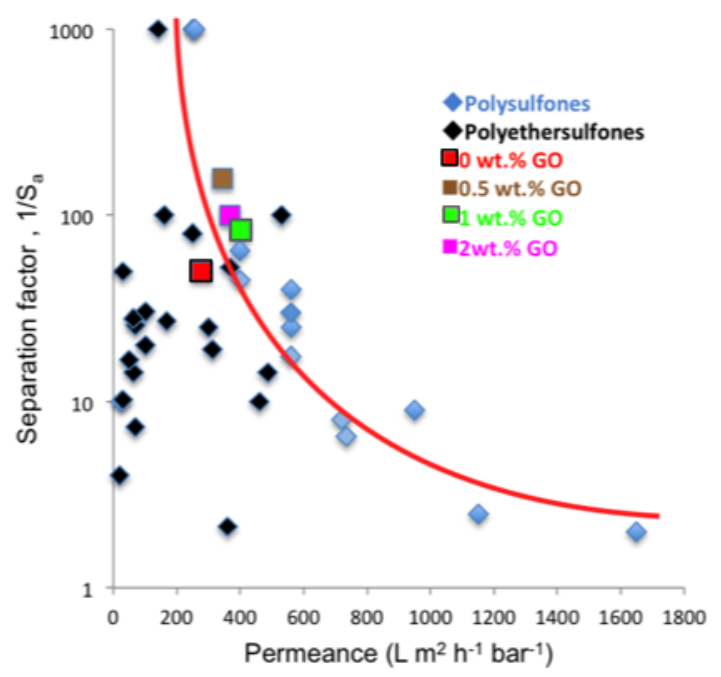

Figure 3. Separation-permeance trade-off for ultrafiltration membranes using BSA as model protein

The mechanical stability of GO incorporated PES membranes was evaluated from stressstrain curves in Figure 2d. The values of stress and strain at break were maximum for $1 \mathrm{wt} \% \mathrm{GO}$. Duan et al.[11] performed similar studies for graphene-immobilized enzyme and PES membranes prepared from solutions in dimethylacetamide and obtained similar results, but with permeation lower than $185 \mathrm{~L} \mathrm{~m}^{-2} \mathrm{~h}^{-1} \mathrm{bar}^{-1}$.

\section{CONCLUSIONS}

GO/PES ultrafiltration membranes were successfully fabricated from solutions in $\mathrm{DMF} /[\mathrm{EMIM}] \mathrm{DEP}$. The modified membranes exhibited enhanced water permeance, due to high hydrophilicity and thin skin layer, confirmed by FESEM, MWCO of $34 \mathrm{~kg} / \mathrm{mol}$ and improved mechanical properties. The positions of the GO/PES membranes are just above the trade-off curve reported in the literature for PES and polysulfone membranes.

\section{ACKNOWLEDGMENTS}

This work was sponsored by King Abdullah University of Science and Technology (KAUST). The authors thank Dr. Mahendra Kumar (KAUST) for his assistance in GO synthesis and Prof. Mainak Majumder for discussions in the early stage of this work.

\section{REFERENCES}


1. S. Livazovic, Z. Li, A.R. Behzad, K.V. Peinemann and S.P. Nunes, J. Membr. Sci. 490, $282(2015)$.

2. $\quad$ D. Kim, O.R. Salazar and S.P. Nunes, Green Chemistry 18, 5151 (2016).

3. $\quad$ D. Kim, N.L. Le and S.P. Nunes, J. Membr. Sci. 520, 540 (2016).

4. D. Kim, N. Moreno and S.P. Nunes, Polym. Chem. 7, 113 (2016).

5. D.Y. Xing, N. Peng and T.-S. Chung, J. Membr. Sci. 380, 87 (2011).

6. P. Madhavan, R. Sougrat, A.R. Behzad, K.-V. Peinemann and S.P. Nunes, J. Membr. Sci. 492, 568 (2015).

7. S. Chisca, M. Torsello, M. Avanzato, Y. Xie, C. Boi and S.P. Nunes, Polymer.

8. $\quad$ B. Van Der Bruggen, J. Appl. Polym. Sci. 114, 630 (2009).

9. A. Akbari, P. Sheath, S.T. Martin, D.B. Shinde, M. Shaibani, P.C. Banerjee, R. Tkacz, D. Bhattacharyya and M. Majumder, Nat. Commun. 7 (2016).

10. J. Zhu, J. Wang, J. Hou, Y. Zhang, J. Liu and B. Van der Bruggen, J. Mater. Chem. A 5, 6776 (2017).

11. L. Duan, Y. Wang, Y. Zhang and J. Liu, Appl. Surf. Sci. 355, 436 (2015).

12. J. Zhu, M. Tian, J. Hou, J. Wang, J. Lin, Y. Zhang, J. Liu and B. Van Der Bruggen, J. Mater. Chem. A 4, 1980 (2016).

13. D.R. Dreyer, S. Park, C.W. Bielawski and R.S. Ruoff, Chem. Soc. Rev. 39, 228 (2010).

14. W. HUMERS and R. Offeman, J. Am. Chem. Soc. 80, 1339 (1958).

15. A. Mehta and A.L. Zydney, J. Membr. Sci. 249, 245 (2005). 